\title{
Documentos
}

\section{El papel de la radioterapia en el tratamiento del cáncer de ovario: revisión de la literatura.}

\author{
Benjamín Bianchi G. ${ }^{1}$, Gonzalo Silva P. ${ }^{2}$, Claudia Carvajal C. ${ }^{2}$, Alejandro Santini B. ${ }^{3}$ \\ ${ }^{1}$ Residente, Programa de Radioterapia Universidad de los Andes, Instituto Nacional del Cáncer. ${ }^{2}$ Interno, Escuela de \\ Medicina, Universidad de los Andes. ${ }^{3}$ Oncólogo-Radioterapeuta, Instituto Nacional del Cáncer. Santiago, Chile.
}

\section{RESUMEN}

Múltiples revisiones han estudiado el potencial coadyuvante en la radioterapia (RT) y quimioterapia (QT) en el tratamiento del cáncer de ovario, pero no se ha comparado adecuadamente la efectividad de la RT abdominal total (RTAT) versus nuevas drogas de QT. Tanto la cirugía como la QT previas a la RT aumentan la sobreviva total y libre de enfermedad, ya que radiosensibilizan al tumor favoreciendo su destrucción. Las distintas técnicas de RTAT no han demostrado diferencias significativas en la sobrevida ni en el control de la enfermedad, pero la RTAT de campos abierto si arrojó menor toxicidad tardía. En la actualidad nuevas técnicas como la IMRT (radioterapia de intensidad modulada) han comenzado a utilizarse con excelentes resultados. A pesar de los avances, muchos de estos pacientes desarrollan recurrencia intraabdominal, quedando fuera del rango terapéutico para QT y en dicho momento la RT puede ser utilizada también en forma paliativa con dosis grandes y únicas, cuidando la aparición de posibles complicaciones. Es probable que en el futuro se reintegre la RTAT en el tratamiento del cáncer de ovario de acuerdo a los hallazgos en el acto operatorio. Finalmente la RT/QT concomitantes, han demostrado su efecto sensibilizador sinergista y es esperable que con el tiempo se sigan integrando nuevas drogas con distintas interacciones como los taxanos y moléculas target. El tratamiento del cáncer de ovario sigue siendo esencialmente quirúrgico y con quimioterapia adyuvante, sin embargo también la radioterapia con quimioterapia han demostrado su efecto adyuvante. A pesar de ser una alternativa poco explorada en la actualidad es necesario estudiar prospectivamente el promisorio resultado de las nuevas técnicas de radioterapia en este tipo de pacientes.

\section{PALABRAS CLAVE: Cáncer de ovario, radioterapia, quimioterapia, revisión de la literatura}

\section{SUMMARY}

Several studies have reviewed the potential coadjuvant of Radiotherapy (RT) and chemotherapy in the treatment of ovary cancer. However the effectiveness of total abdomen RT (RTAT) versus new QT drugs have not been adequately compared. Both surgery and QT prior to RT increase total and freedom from the disease since tumors become radiosensitive, thus favoring destruction. Diverse techniques such as IMRT (Intensity modulated radiation therapy) are being used with excellent results. Despite these advances, many of the patients develop intrabdominal recurrence leaving them out of the therapeutic scope/range for QT. RT may then be used as a palliative in big unique cases watching possible complications. RTAT may eventually be restarted in the treatment of ovary cancer depending on the findings during surgery. Finally, RT/QT concomitants have shown their effect in sensitivity and synergy and it is expected that new drugs with different interactions such as taxan and target molecules are added. Treatment of ovarian cancer remains essentially 
surgical and with chemotherapy, but both radio and chemotherapy has demonstrated their adjuvant effect. Despite being an unexplored alternative is necessary to study prospectively the promising results of new techniques of radiation therapy in these patients.

\section{KEY WORDS: Ovary cancer, radiotherapy, chemotherapy, literature review}

\section{INTRODUCCIÓN}

El cáncer de ovario $(\mathrm{CO})$ es actualmente la primera causa de muerte por cáncer ginecológico en Estados Unidos, con 21.000 casos nuevos por año, superando al cáncer de endometrio y cérvix juntos (1). El notorio aumento de su tasa de mortalidad durante los últimos 15 años demuestra que el $\mathrm{CO}$ es un problema de salud importante en la actualidad y el futuro. La piedra angular de la terapia contra el CO continúa siendo la cirugía, que es el tratamiento primario en la gran mayoría de las pacientes. Su extensión y tipo de cirugía ha sido establecido (2), y los objetivos principales de la terapia quirúrgica consisten en tres puntos fundamentales: estatificar, establecer un diagnóstico histológico y reducir al máximo el volumen tumoral (debulking).

Sin embargo, los resultados de la cirugía como tratamiento exclusivo no han sido del todo satisfactorios, y es por ello que otros tratamientos complementarios han sido aplicados no exentos de controversias, como la debida a la aparición de la quimioterapia (QT) con cisplatino en la década de los setenta y con taxanos en los noventa. A pesar de ello, la mortalidad del $\mathrm{CO}$ ha continuado su ascenso con un aumento del $18 \%$ sólo en la última década (3). Pese a los esfuerzos, se ha visto que entre los pacientes en que se ha obtenido una respuesta completa comprobada por second-look, el $50 \%$ recae y fallece como consecuencia de la enfermedad. De acuerdo a Makatsosis y cols (4), con los nuevos regímenes de QT de platinos mas taxanos se obtiene en promedio $50 \%$ de respuestas completas clínicas de las cuales solo la mitad tendrán una respuesta completa patológica demostrada por second-look, y de ellos solo el $20 \%$ llegarán a sobrevivir a los 5 años. En suma, solo 5 de cada 100 pacientes estarían "curados" a los 5 años, por lo que el CO puede ser tratado con QT pero no necesariamente curado con dicho tratamiento.

A diferencia de la QT, la radioterapia (RT) ha demostrado ser un tratamiento curativo en la enfermedad microscópica o de pequeño volumen, pero su papel aún no ha sido claramente establecido. El rol de la RT abdominal total (RTAT) como consolidador luego de la QT o tratamiento de salvataje, permanece en controversia. Además, el surgimien- to de la nueva QT ha mantenido a la RTAT sin ser utilizada en forma importante durante las dos últimas décadas, sin embargo, en los últimos años ha habido un resurgimiento de la RT como parte integral de un tratamiento multimodal.

Si miramos el comportamiento histórico de las técnicas más utilizadas en oncología podemos ver el ejemplo de la RT en el cáncer de mama. En un principio la RT se reservaba exclusivamente para después de una mastectomía, pues en la década de 1980 algunos trabajos demostraron que ésta disminuía la sobrevida de las pacientes debido a toxicidad cardiovascular. Pero luego de varios años la RT moderna demostró un aumento de la sobrevida como consecuencia de un mayor control local sin provocar toxicidad cardiovascular (5), lo que nos lleva a pensar que tal vez la historia se repita con la $\mathrm{RT}$ y el $\mathrm{CO}$, mientras se vaya depurando la técnica y se realicen indicaciones cada vez más precisas. No existen en la actualidad estudios importantes que comparen la RTAT versus la QT moderna. Tal como lo planteó el Dr. Ozols en el clásico libro de Vincent de Vita en 1997: "...aunque los resultados de los trabajos retrospectivos no muestran una clara diferencia de un tratamiento sobre el otro, los prejuicios de los médicos son grandes."

\section{RTAT ADYUVANTE LUEGO DE LA CIRUGÍA}

Durante las décadas de 1960 y 1970 la RT fue un tratamiento fundamental como complemento de la cirugía en el $\mathrm{CO}$. El tratamiento locorregional que ofrece la RT es efectivo, debido a que el CO es una enfermedad peritoneal que aun en estadios avanzados se mantiene recluido al abdomen en forma intraperitoneal, en el retroperitoneo y los ganglios de la pelvis. Es por ello que los primeros trabajos incluían solo RT sobre la pelvis, pero se ha demostrado que esta forma de tratamiento era insuficiente. Remberger y cols (6), analizaron 137 autopsias de pacientes que fallecieron por $\mathrm{CO}$ demostrando que no más del $56 \%$ de ellos tenían una enfermedad limitada al peritoneo. Luego otro trabajo comprobó que solo en el $2 \%$ de los pacientes se encontraron metástasis fuera del abdomen cuando no hay enfermedad abdominal (7).

Con el advenimiento de las nuevas drogas en 
QT, el papel de la RT ha ido disminuyendo, sobre todo luego de lo publicado por el grupo del MD Anderson que comparó el melfalan con RTAT (8). Se incluyeron 108 pacientes con estadio I-II que fueron randomizados entre 12 ciclos de melfalan (mono QT) y RTAT. La sobrevida actuarial a 2 años, para los estadios I y II fue respectivamente $85 \%$ y $55 \%$ para RTAT, y $90 \%$ y $58 \%$ para la QT, la toxicidad fue mayor para la RTAT y el costo del melfalan fue francamente menor. Posterior a su publicación el trabajo fue duramente criticado por problemas técnicos con respecto a la no irradiación del diafragma, la baja dosis a nivel hepático y renal, el desbalance entre las dos ramas y el cortísimo seguimiento. Hoy por hoy la QT no es la misma, su toxicidad y costo se ha hecho cada vez mayor mientras la RT ha disminuido notoriamente su toxicidad (9).

Ozols y cols (10), demostraron que con RT la sobrevida a 10 años es entre 38 y $62 \%$ cuando la enfermedad residual postoperatoria es menor de 2 $\mathrm{cm}$ y de 0 a $10 \%$ cuando es mayor de $2 \mathrm{~cm}$. Este trabajo comprobó además que el volumen tumoral postoperatorio es el factor determinante en el resultado del tratamiento radiante del $\mathrm{CO}$. Fue en base a este conocimiento que Dembo y cols del Princess Margaret Hospital de Canadá (PMH) (11), desarrollaron el esquema de clasificación de riesgo para las pacientes con $\mathrm{CO}$, que es la base para la decisión terapéutica de aquellas pacientes (Tabla I). Los resultados de RTAT obtenidos en PMH evidencian la diferencia del $\mathrm{CO}$ al clasificarlo en distintos grupos de riego (alto e intermedio), en donde existe una diferencia significativa entre ambos grupos, tanto para sobrevida global (SG) como para sobrevida libre de enfermedad (SLE). En el grupo de riesgo intermedio la SG fue de $68 \%$ a 10 años de seguimiento y la SLE fue de $67 \%$ a 10 años. En el grupo de alto riesgo en cambio, tanto la SG como la SLE a 10 años fue de $17 \%$ ( $p<0,0005)$. Sin embargo, en el grupo de alto riesgo, a 5 años de seguimiento, si existe diferencia significativa entre SG y SLE, con $32 \%$ y $23 \%$ respectivamente.

Sedlasek y cols (12), apoyaron los resultados del PMH con una revisión de 12 trabajos (casi 700 pacientes) que demostró que la sobrevida a 10 años era del $36 \%$ para enfermedad residual microscópica y del $6,8 \%$ para la macroscópica. Reinfuss y cols (13), incluyeron 345 pacientes con CO no tratados con QT y de acuerdo a la enfermedad residual comprobaron una sobrevida del $70 \%$ a los 5 años para la enfermedad residual microscópica (citología positiva), del $40 \%$ para la residual menor de $3 \mathrm{~cm}$, y del 2,8\% cuando era mayor de $3 \mathrm{~cm}$. Pero además del volumen, los resultados también dependen de la técnica de RT utilizada y su extensión. La sobrevida a 5 años fue del $32,5 \%$ cuando se trató solo el abdomen, del $32,9 \%$ cuando se trató solamente la pelvis y del $49 \%$ cuando se trató el abdomen más sobreimpresión en la pelvis.

En la Tabla II se resume los resultados de las principales series de RTAT como tratamiento postoperatorio exclusivo para enfermedad residual mínima (estadios I-III). A nivel nacional, Hepp y cols (14), en una serie de 60 pacientes en etapas I-III obtuvieron resultados similares con una toxicidad aceptable.

Tabla I

\section{CLASIFICACIÓN DE RIESGO PARA PACIENTE CON CÁNCER DE OVARIO}

\begin{tabular}{cclll}
\hline Estadio & $\begin{array}{c}\text { Residuo } \\
\text { post-quirúrgico }\end{array}$ & Grado 1 & Grado 2 & Grado 3 \\
\hline I & 0 & Riesgo bajo & Riesgo medio & Riesgo medio \\
II & 0 & Riesgo medio & Riesgo medio & Riesgo medio \\
II & $<2 \mathrm{~cm}$ & Riesgo medio & Riesgo medio & Riesgo alto \\
III & 0 & Riesgo medio & Riesgo alto & Riesgo alto \\
III & $<2 \mathrm{~cm}$ & Riesgo medio & Riesgo alto & Riesgo alto \\
\hline
\end{tabular}

Según las indicaciones del PMH aquellas pacientes con bajo riesgo (estadio I sin enfermedad residual y grado histológico 1) no necesitan tratamiento complementario, mientras las pacientes con riesgo intermedio deben tratarse con RTAT, y las con riesgo elevado con QT (estadio III y IV, grado histológico 2 o 3, y enfermedad residual mayor de $2 \mathrm{~cm}$ ). 
Tabla II

RESULTADOS DE RADIOTERAPIA ABDOMINAL TOTAL PARA ENFERMEDAD RESIDUAL MÍNIMA

\begin{tabular}{|c|c|c|c|c|}
\hline Autor & Estadio & Volumen residual & Respuesta & $\begin{array}{l}\text { Sobrevida } \\
\text { media }\end{array}$ \\
\hline Reinfuss $^{13}$ & II-III & $\begin{array}{l}\text { Enfermedad residual micro } \\
\text { Enfermedad residual macro }(<3 \mathrm{~cm}) \\
\text { Enfermedad residual macro }(>3 \mathrm{~cm})\end{array}$ & $\begin{array}{l}70 \% \text { a } 5 \text { años } \\
40 \% \text { a } 5 \text { años } \\
2,8 \% \text { a } 5 \text { años }\end{array}$ & 5 años \\
\hline Hepp ${ }^{14}$ & I y |II & & $\begin{array}{l}\text { ST } 55 \% \\
\text { Etapa I-II } 66 \% \\
\text { Etapa III } 35 \%\end{array}$ & 5 años \\
\hline & II y III & $<2 \mathrm{~cm}$ & SV 10 años $42 \%$ & 6 años \\
\hline Goldberg $^{15}$ & II y III & Riesgo intermedio & SVLE 10 años 79\% & $>5$ años \\
\hline Dembo $^{16}$ & $\begin{array}{l}\text { II } \\
\text { III }\end{array}$ & $\begin{array}{l}<2 \mathrm{~cm} \\
<2 \mathrm{~cm}\end{array}$ & $\begin{array}{l}\text { SV } 5 \text { años } 78 \% \\
\text { SV } 5 \text { años } 43 \%\end{array}$ & $\begin{array}{l}>5 \text { años } \\
>5 \text { años }\end{array}$ \\
\hline Fuller ${ }^{17}$ & I-III & $<2 \mathrm{~cm}$ & SVLE 10 años $71 \%$ & $>10$ años \\
\hline Petereit ${ }^{18}$ & I-III & Riesgo intermedio & SVLE 9 años $62 \%$ & > 9 años \\
\hline Weiser ${ }^{19}$ & II-III & No & SV 10 años 59\% & >10 años \\
\hline
\end{tabular}

SV: sobrevida, SVLE: sobrevida libre de enfermedad.

En la literatura existen muy pocos trabajos, realizados en forma óptima, y que comparen la RTAT con QT. Sólo se han reportado 5 trabajos randomizados donde sólo en uno se utiliza cisplatino. Dembo (16), mostró una mejoría significativa cuando compara RTAT contra RT pelviana +/- clorambucil. Los demás trabajos no demuestran una diferencia significativa entre sus ramas como se ve en la Tabla III.
El trabajo del PMH de Canadá comparó en 147 pacientes dos ramas de tratamiento: RTAT 2250 cGy/10 fracciones con técnica de franja móvil versus RT pelviana 4500 cGy/20 fracciones seguida de clorambucil por $6 \mathrm{mg} / \mathrm{d}$ por dos años (11) Para las pacientes con mínima enfermedad residual (menos de $2 \mathrm{~cm}$ ) se comprobó una ventaja estadísticamente significativa a favor de la RTAT $(64 \%$ vs

Tabla III

RESULTADOS DE TRABAJOS RANDOMIZADOS QUE COMPARAN RADIOTERAPIA ABDOMINAL TOTAL CON QUIMIOTERAPIA

\begin{tabular}{|c|c|c|c|c|}
\hline Autor & Estadio & $\begin{array}{c}\text { Ramas } \\
\text { de tratamiento }\end{array}$ & $\begin{array}{c}\mathrm{N}^{\circ} \\
\text { de pacientes }\end{array}$ & Resultados \\
\hline \multirow[t]{2}{*}{ Dembo $^{16}$} & I-III (D) & RTAT & 76 & SV 10 años 64\% \\
\hline & & RT pelviana +/- clorambucil & 71 & SV 10 años $40 \% p=.0007$ \\
\hline \multirow[t]{3}{*}{ Klaassen ${ }^{20}$} & IA,IB, grado 3 & $\mathrm{RT}$ pelviana $+\mathrm{P} 32$ & 44 & SV 5 años $66 \%$ \\
\hline & & RT pelviana + melfalan & 106 & SV 5 años $61 \%$ \\
\hline & IC-II (D) & RTAT & 107 & SV 5 años $62 \% p=N S$ \\
\hline \multirow[t]{2}{*}{ Smith ${ }^{21}$} & 1 & $\begin{array}{l}\text { RTAT vs } \\
\text { melfalan }\end{array}$ & 42 & $\begin{array}{l}\text { SV } 2 \text { años } 85 \% \\
\text { SV } 2 \text { años } 90 \%\end{array}$ \\
\hline & II & $\begin{array}{l}\text { RTAT vs } \\
\text { melfalan }\end{array}$ & 66 & $\begin{array}{l}\text { SV } 2 \text { años } 55 \% \\
\text { SV } 2 \text { años } 58 \%\end{array}$ \\
\hline \multirow[t]{2}{*}{ Sell $^{22}$} & 1 & RTAT vs & 60 & SV 4 años $63 \%$ \\
\hline & & Rt pelviana + melfalan & 58 & SV 4 años $55 \% p=N S$ \\
\hline \multirow[t]{2}{*}{ Chiara $^{23}$} & $|-I|$ & Cisplatino + Ciclofofamida vs & 44 & SV 5 años $73 \% p=N S$ \\
\hline & & RTAT & 25 & SV 5 años $68 \%$ \\
\hline
\end{tabular}

SV: sobre vida 
$40 \% ; p=0,0007$ ), pero no se vio diferencia para los pacientes con enfermedad residual mayor de $2 \mathrm{~cm}$.

El estudio prospectivo randomizado realizado por el grupo cooperativo del noroeste de Italia causó grandes repercusiones para pacientes en estadio temprano con enfermedad de alto riesgo (estadío IA o IB pobremente diferenciados o estadio IC o II) (22). Se realizaron dos ramas, una de QT cisplatino mas ciclofosfamida por 6 ciclos, y la otra de RTAT $(42,2$ Gy a la pelvis y 30,2 Gy a todo el abdomen. Los resultados de SVLE a 5 años fue $73 \%$ para QT vs $60 \%$ RT $(p=0,3)$ y la sobrevida global (SG) a 5 años fue de $73 \%$ para QT vs $68 \%$ para RT $(p=0,7)$. A primera vista dichos resultados descartan el empleo de RT, pero al analizarlo exhaustivamente veríamos que no solo fue cerrado prematuramente por incumplimiento del protocolo, sino que además el $15 \%$ de los pacientes no tenían una estadificación quirúrgica. Además había 44 pacientes en la rama de QT y 25 en la de RT, ¿fueron los sujetos en estudio realmente randomizados? Por último, la dosis empleada diariamente en la técnica de tratamiento radiante es alta (1,8 Gy), y por otro lado, se utilizó una energía de $18 \mathrm{Mev}$, lo que es demasiado alta y subdosifica el peritoneo parietal superficial. Debido a todo esto podemos afirmar que de este estudio no puede rescatarse una conclusión valedera dado sus sesgos técnicos y metodológicos (23).

En suma, no tenemos la información suficiente para decir que un tratamiento sea más efectivo que el otro. No se han hecho comparaciones con nuevas drogas como los taxanos y además cuando se analiza la evidencia para la elección de los trata- mientos la RT sigue utilizándose en pacientes que no son candidatas a QT luego de una cirugía de citoreducción óptima $(24,25,26)$. Veremos, a continuación el posible papel de esta forma de tratamiento, en el marco de un tratamiento multimodal del CO.

\section{RT DE CONSOLIDACIÓN LUEGO DE CIRUGÍA Y QUIMIOTERAPIA}

En forma aislada, tanto la RTAT como la QT han fallado en demostrar resultados alentadores a largo plazo para los pacientes de alto riesgo. En base a eso ambas técnicas se han utilizado en forma conjunta, como es el caso de la RT luego de cirugía y QT. En la Tabla IV se analizan algunos de estos trabajos.

Haie y cols (31) del Gustave Roussy, analizaron el papel de la RTAT luego de second-look comprobando que uno de los factores pronósticos más importantes era la presencia de enfermedad residual en el second-look. La sobrevida libre de enfermedad fue de 16 y $68 \%$ respectivamente. Pickel y cols (32) randomizaron pacientes con $\mathrm{CO}$ sin enfermedad residual luego de la cirugía en dos ramas: una con QT sola (cisplatino y epirrubicina por 6 series) y otra con el agregado de RTAT luego de la misma QT. La sobrevida libre de enfermedad a 5 años fue de $26 \%$ para QT sola y de $49 \%$ para RTQT ( $p=0,013$ ), la sobrevida total a 5 años fue de $33 \%$ para QT y $59 \%$ para QTRT $(p=0,029)$, la diferencia fue mayor para los pacientes estadio III y la toxicidad fue aceptable. Por su parte Dinniwell y cols (33), obtuvieron una sobrevida total del $92 \%$

\section{Tabla IV}

ANÁLISIS DEL ROL DE LA RADIOTERAPIA DE CONSOLIDACIÓN LUEGO DE CIRUGÍA Y QUIMIOTERAPIA

\begin{tabular}{|c|c|c|c|}
\hline Autor & QT & $\mathrm{N}^{\circ}$ pacientes & Resultado \\
\hline \multirow[t]{2}{*}{ Lederman $^{27}$} & $(\mathrm{Cf}+\mathrm{Dox}+\mathrm{CP}) \times 6+\mathrm{RTAT}$ & 86 & $\begin{array}{l}\text { SVM 5,7 a } \\
\text { SVLE5 42,6 \% }\end{array}$ \\
\hline & RTAT & 120 & $\begin{array}{l}\text { SVM 2,4 a } \\
\text { SVLE5 } 21,6 \%\end{array}$ \\
\hline Thomas $^{28}$ & $(C P+C f)+$ RTAT & 105 & $\begin{array}{l}\text { SVT5 76\% (SL-) } \\
66 \% \text { (enf m) } \\
22,4 \% \text { (enf M) }\end{array}$ \\
\hline Randal $^{29}$ & $(\mathrm{CP}+\mathrm{Cf}) \times 3+\mathrm{RTAT}(\mathrm{HF})$ & 42 III (IIIC 93\%) & $\begin{array}{l}\text { SVLE } 24 \mathrm{~m} \\
\text { SVT } 48 \mathrm{~m}\end{array}$ \\
\hline Goldhirisch ${ }^{30}$ & $\begin{array}{l}(\mathrm{CP}+\text { Melf }) \times 6 \\
(\mathrm{CP}+\text { Melf }) \times 6+\text { RTAT }\end{array}$ & & $\begin{array}{l}\text { SVLE } 49 \% \\
\text { SVLE } 89 \%\end{array}$ \\
\hline
\end{tabular}

QT: (quimioterapia), Cf (ciclofosfamida), Dox (doxorubicina), CP (cisplatino), Melf (melfalan), HF (hiperfraccionamiento), SVM (sobrevida media), SVLE (sobrevida libre de enfermedad), SVT (sobrevida total), SL (second look). 
en pacientes sometidos a RT abdominal luego de una resección óptima y QT con paclitaxel y platino con una toxicidad aceptable. Biete y cols (34), obtuvieron una sobrevida libre de enfermedad de $60 \%$ y una sobrevida total de $70 \%$ en pacientes sometidos a RT abdominal luego de una citorreducción óptima más QT con platinos, con un riesgo aceptable de toxicidad aguda. En definitiva todos estos trabajos permiten afirmar que al menos en un grupo determinado de pacientes, la RT de consolidación tiene un papel importante con un aumento de la sobrevida libre de enfermedad y de la sobrevida total.

\section{PAPEL DE LA RT EN LOS PACIENTES REFRAC- TARIOS A LA PRIMERA LÍNEA DE TRATAMIEN- TO QUIMIOTERÁPICO (PLATINOS Y TAXANOS)}

Frecuentemente la literatura nos impide distinguir con claridad entre aquel tratamiento combinado planificado, que se indica a los pacientes que no tienen evidencia de enfermedad con elevado riesgo de recurrencia, de aquel tratamiento de salvataje que se realiza en pacientes con enfermedad patológicamente demostrada. Los tratamientos combinados permiten modificar o reducir la intensidad de la QT previniendo la toxicidad. La RT debe agregarse en forma precoz para evitar la resistencia cruzada entre esta forma de tratamiento y el cisplatino. La respuesta al tratamiento de rescate también depende en gran medida del volumen tumoral residual. Dicha respuesta ha demostrado ser del alrededor de un $30 \%$ para los pacientes con enfermedad residual microscópica y del $10 \%$ cuando la misma es mayor (32), sin embargo, es difícil saber cual es el porcentaje de respuesta a largo plazo dado que algunos autores la calculan desde el inicio del tratamiento y otros desde el inicio del tratamiento de rescate.

En la publicación de Reddy y cols (35), trataron 44 pacientes que habían recaído luego de la QT más RTAT con técnica de campos abiertos, 25 Gy y boost sobre los lugares con enfermedad más voluminosa; la sobrevida para los pacientes con enfermedad microscópica fue de $42 \%$ a los 4 años.

Sedlaseck y cols (12), reportaron recientemente resultados similares para los pacientes refractarios a QT intensiva con platino, mientras Baker y cols (36) también comprobaron que los pacientes con enfermedad limitada a la pelvis tienen mejor resultado que los que tienen diseminación a abdomen, incluso cuando la enfermedad es voluminosa.

Por su parte Cmelak y cols (37), reportaron la experiencia de la Universidad de Stanford con RTAT en 41 pacientes con enfermedad persistente o recurrente luego de una cirugía de debulking, QT y second-look. La dosis media entregada a la totalidad del abdomen y la pelvis, con la técnica de campos abiertos fue de 28 Gy y 38 Gy respectivamente. Los pacientes con enfermedad estadio I-III con enfermedad residual menor de $1,5 \mathrm{~cm}$ tuvieron una sobrevida actuaria libre de enfermedad a 10 años del $50 \%$, sin embargo, un $30 \%$ de los pacientes no lograron completar el tratamiento por la toxicidad aguda. Cabe destacar eso si que solo en un paciente ocurrió toxicidad tardía grave (G IV) con obstrucción intestinal.

En un intento de mejorar la tolerancia de los tratamientos, varios autores han intentado utilizar fraccionamientos no convencionales para el tratamiento de salvataje.

Eifel y cols (38), del MD Anderson reportaron su experiencia con fracciones de 1 Gy dos veces por día, llegando a 30 Gy totales a todo el abdomen con una interrupción planificada a los 15 Gy. Lamentablemente sus resultados fueron desalentadores ya que obtuvieron una sobrevida de $10 \%$ a los 3 años para los pacientes con enfermedad residual microscópica.

Fein y cols (39), de la Universidad de Florida, también hiperfraccionaron el tratamiento radiante pero bajando la dosis por fracción a 0,8 Gy hasta 30,4 Gy sin interrupciones. Ellos reportaron una sobrevida libre de enfermedad a 5 años de $18 \%$ con sobrevida total de $28 \%$ y a pesar de haber recibido entre 2 y 4 laparotomías (promedio 2,6) y 6 a 28 ciclos de QT (promedio 12) antes de la RT, solo 2 pacientes requirieron interrupción del tratamiento (7\%). Además 4 pacientes (14\%) debieron operarse por una oclusión intestinal donde 2 tenían enfermedad residual. En la Tabla $V$ se resumen algunos de los trabajos de RT de salvataje en pacientes refractarios a la QT.

\section{ASPECTOS TÉCNICOS DE LA RTAT}

Existen varias técnicas de RTAT, ellas la llamada de franja móvil (FM) o moving strip y la de campos abiertos (CA) con sus modificaciones. Independiente de la técnica utilizada, la RTAT debe hacerse con equipo de megavoltage y con una distancia fuente-piel de 100 a $180 \mathrm{~cm}$ para lograr cubrir el gran volumen que va desde el fondo de la pelvis al diafragma (43).

En la simulación se debe comprobar que el borde superior del campo quede por lo menos $1,5 \mathrm{~cm}$ por arriba del diafragma en la expiración. Lateralmente el borde debe ir más allá del reborde peritoneal, el cual debe ser meticulosamente establecido debido a que en decúbito el repliegue peritoneal lateral se desplaza lateralmente (Figura 1) (44). Con ambas técnicas se realiza una sobreimpresión, a través de cuatro campos con la técnica de la caja sobre la pelvis. 
Tabla V

RESULTADOS DE LA RADIOTERAPIA ABDOMINAL TOTAL DE SALVATAJE RESPECTO A LA ENFERMEDAD RESIDUAL LUEGO DE CIRUGÍA Y QUIMIOTERAPIA *

\begin{tabular}{|c|c|c|c|c|}
\hline Autor & $\mathrm{n}$ & $\begin{array}{c}\text { SV pacientes con } \\
\text { enfermedad residual } \\
\text { micro }\end{array}$ & $\begin{array}{c}\text { SV pacientes con } \\
\text { enfermedad residual } \\
\text { macro }\end{array}$ & SV total \% \\
\hline Sedlacek $^{12}$ & 27 & -- & -- & $15 \% 5$ años \\
\hline Baker $^{36}$ & 51 & 42 & 11 & $32 \% 4$ años \\
\hline Cmelak $^{37}$ & 41 & 40 & 0 & $47 \% 5$ años \\
\hline Eifel $^{38}$ & 37 & 10 & 0 & $24 \% 3$ años \\
\hline Fein $^{39}$ & 28 & 27 & 18 & $21 \% 5$ años \\
\hline Bolis 40 & 26 & 50 & 25 & $34 \% 3$ años \\
\hline Kurten 41 & 43 & 66 & 5 & ---- \\
\hline Schray ${ }^{42}$ & 53 & 34 & 28 & $30 \% 3$ años \\
\hline
\end{tabular}

* Sobrevida (SV) a 5 años.
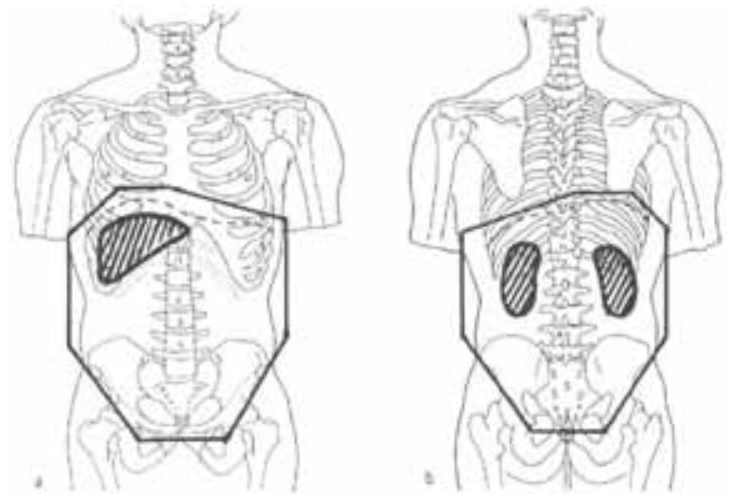

Figura 1. Campos de tratamiento radiante en el cáncer de ovario. Reproducción autorizada por John Wiley e hijos a través del servicio Copyright Clearance Center.

Fuks y cols (45) en 1975, calcularon tanto la Nominal Single Dose (NSD) como los Time-dose fractionations (TDF) e indicaron que para las dos técnicas ambos números son esencialmente el mismo, lo que significa que radiobiológicamente son iguales. En la técnica de FM se realizan bandas de 2,5 $\mathrm{cm}$ desde el extremo superior del campo (diafragma) hasta la parte inferior (pelvis), tanto en el frente como en la espalda. Se comienza por el extremo superior con la irradiación de una franja diaria y se van agregando más de a una hasta llegar a 4 , quedando entonces una franja mayor integrada por 4 franjas menores y que miden $10 \mathrm{~cm}$ de ancho. Luego se va bajando de a una franja hasta llegar al extremo inferior. Con esta técnica la dosis tumoral es de 28 Gy en el plano medio del abdomen, entre- gados en 8 fracciones durante 10-12 días. Con la técnica de CA la totalidad del abdomen se irradia diariamente, entregándose una dosis de 30 Gy en 5 - 6 semanas, con una fracción diaria de 1,5 Gy. El hígado es rutinariamente bloqueado en los campos anterior y posterior mientras que los riñones solamente por el campo posterior.

Martínez y cols (46), desarrollaron algunas modificaciones en la técnica de CA con la llamada técnica de las tres fases. En la primera fase la totalidad del abdomen se irradia hasta $10 \mathrm{~Gy}$, luego se colocan bloqueos de $100 \%$ sobre los riñones por el campo posterior y a los 15 Gy se bloquea con el $50 \%$ el hígado por los campos anterior y posterior hasta llegar a 30 Gy. Durante la segunda fase la parte media del diafragma, los ganglios lumboaórticos y la pelvis son tratados con dos campos anteroposteriores llegándose hasta 42 Gy. En la tercera fase se trata solamente la pelvis completándose $51 \mathrm{~Gy}$.

Dos estudios prospectivos randomizados comparan las dos técnicas clásicas de RTAT. Fazekas y Mayer (47) contrastaron el tratamiento de 30 Gy en 10 días utilizando la técnica de FM y el de 40 Gy en 56 días con campos abiertos sin encontrar diferencias en la sobrevida ni en el control de la enfermedad.

Recientemente Dembo y cols (48), compararon también ambas técnicas de irradiación abdominal. En ambas situaciones se aplicó una sobreimpresión sobre la pelvis de 22,5 Gy en 10 aplicaciones y tanto la sobrevida total, como la sobrevida libre de enfermedad y la tasa de recaídas fue idéntica entre ambas ramas, pero hubo menos efectos tóxicos tardíos con los campos abiertos: obstrucción intestinal en el $6 \%$ de los pacientes en la rama de $\mathrm{FM}$ versus un $1,2 \%$ en la rama de $C A$. 
Debido a este resultado, la simplicidad de la técnica y la menor duración del tratamiento (6-7 semanas versus 11-13 semanas) en el PMH se adoptó la técnica de CA como estándar. En la actualidad varios centros han comenzado a utilizar técnicas más depuradas de RT, IMRT, Rapadarc, etc., con resultados alentadores y una toxicidad cada vez menor (49).

En la Tabla VI se resumen los principios básicos de la radioterapia abdominal total.

\section{RT PALIATIVA}

No hay duda que la RT tiene un papel muy importante en el tratamiento paliativo de los pacientes con cáncer, pero como mencionamos anteriormente, a pesar de los avances en los tratamientos actuales, más de dos tercios de los pacientes desarrollarán una recurrencia intrabdominal, que en la mayoría de los casos será la causa de su deceso.

\section{Tabla VI}

\section{PRINCIPIOS BÁSICOS DE TRATAMIENTO CON RADIOTERAPIA ABDOMINAL TOTAL}

\section{Principios}

1 La RTAT debe ser realizada solamente en pacientes sin enfermedad macroscópica en el abdomen superior, o no mayor de 2-3 cm en la pelvis.

2 Se debe incluir la totalidad de la cavidad abdominal y se requiere verificación radiológica.

3 Cuando la dosis se limita a 25-28 Gy con un fraccionamiento de 1-1,2 Gy al día no se requiere bloqueo hepático.

4 Se bloquean los riñones para no sobrepasar de 1820 Gy

5 La pelvis debe recibir una sobreimpresión, con un fraccionamiento de 1,8-2 Gy día hasta completar una dosis de 45 Gy.

6 Campos paralelos y opuestos se emplean con una energía suficiente para permitir una variación de la dosis de menos del $5 \%$

7 El campo debe extenderse mas allá de la cresta ilíaca, para asegurar la cobertura de la totalidad de la cavidad peritoneal

8 El borde superior debe sobrepasar con un margen de $2 \mathrm{~cm}$ el diafragma en la expiración.

9 Cuando es un tratamiento de salvataje, y el mismo es postoperatorio (second-look), es importante que el cirujano deje clips metálicos para la localización de las masas mayores.

RTAT: radioterapia abdominal total
Actualmente no hay tratamiento efectivo para aquellos pacientes con una recurrencia abdominal voluminosa cuando se agotaron 1 o 2 líneas de QT (50), pero la RT puede ser utilizada en forma paliativa tanto sobre masas que provocan dolor o compresión de estructuras, como en las responsables de sangrados. Un trabajo interesante del MD Anderson reportó que con el empleo de dosis grandes y únicas de hasta $10 \mathrm{~Gy}$, la respuesta era entre un $55 \%$ y $72 \%$ para 42 mujeres con $\mathrm{CO}$ que tenían sangrado o dolor (51), sin embargo, estas fracciones tan grandes tienen una mayor tasa de complicaciones. Corn y cols (52), trataron 33 pacientes con $\mathrm{CO}$ con criterio paliativo, logrando $51 \%$ de respuestas y $79 \%$ de reducción de la sintomatología; en este trabajo la duración media de la paliación fue de 4 meses.

\section{¿HACIA DONDE VA LA RT EN EL CÁNCER DEL OVARIO?}

Recientemente se publicó un excelente trabajo donde se analiza con detalle cuales son las directivas en el futuro de la RT en el CO (53).

a. QT primaria, cirugía y RT. Esta forma de QT neoadyuvante ha sido revisada por múltiples autores. Schwartz y cols (54), publicaron la experiencia de la Universidad de Yale donde se emplea QT neoadyuvante en base a cisplatino. De los 49 pacientes entrados en el protocolo 41 recibieron tratamiento quirúrgico citorreductor y no se encontró diferencia en la sobrevida total o en la sobrevida libre de progresión respecto al grupo de pacientes tratados de forma convencional. Esta forma de tratamiento, dicen los autores, permite integrar la RTAT de acuerdo a los hallazgos en el acto operatorio.

b. Nuevas técnicas de RT, IMRT. Nuevas técnicas de tratamiento, se están ensayando para la irradiación total del abdomen, fundamentalmente IMRT que ha obtenido una reducción en los efectos indeseables con resultados alentadores tanto en la paliación como en los tratamientos con fines curativos. c. RT concurrente con $Q T$. Se ha demostrado en el laboratorio y en la clínica la sensibilización que existe entre el platino y la RT en varias localizaciones tumorales, a tal extremo, que para varios es el tratamiento de elección $(55,56)$. Silver y cols $(56)$, también han comprobado ese sinergismo entre el platino y la RT para las líneas celulares de laboratorio, que explica como las células mueren con dosis significativamente más bajas, lo que es de gran interés para el manejo clínico. King y cols (57), realizaron un estudio en el cual realizaban RTAT en forma conjunta con cisplatino intraperitoneal previo a la cirugía. El tratamiento presentó una elevada 
toxicidad tanto hematológica como gastrointestinal. Sin embargo, es de destacar que en este trabajo, que data de 1991 no se emplearon ni los factores estimulantes de colonia para superar las leucopenias, ni tampoco se contaba con los nuevos citoprotectores. Recientemente, Dowlatshahi y cols (24), desarrollaron un trabajo piloto donde entregaron 30 Gy en 30 fracciones en forma conjunta con dosis bajas de cisplatino $(5 \mathrm{mg} / \mathrm{m} 2)$ semanal, con un seguimiento medio de 32 meses, la sobrevida proyectada a 5 y 10 años fue de 44 y $35 \%$.

d. Integración con nuevas drogas. Por último se están estudiando las posibles interacciones de nuevas drogas que ya se han demostrado útiles en el tratamiento del CO y que además son excelentes radiosensibilizantes, entre ellos los taxanos y moléculas target (58).

\section{CONCLUSIÓN}

El cáncer de ovario presenta altas tasas de mortalidad y de incidencia en aumento. El acercamiento para un tratamiento efectivo debe ser comandado por la cirugía, cuando la enfermedad esta limitada al abdomen. Sin embargo debemos mejorar más aún los resultados de las terapias adyuvantes, tanto con radioterapia como quimioterapia, ya que los resultados con cirugía sola son desalentadores. La radioterapia adyuvante aun no tiene un papel bien establecido, sin embargo, no hay suficientes argumentos para decir que ningún paciente debe recibirla. Los trabajos originales de las décadas del 80 y 90 muestran beneficios claros y no hay trabajos recientes que digan lo contrario. En la actualidad existen técnicas más modernas que las empleadas en los trabajos originales, con lo cual se puede reducir los efectos adversos y por lo tanto mejorar los índices terapéuticos. Los trabajos modernos al respecto muestran resultados bastante promisorios con intensidad modulada y otras técnicas bien conformadas.

Si bien esta técnica, no es muy popular en nuestro medio, creemos importante iniciar estudios clínicos prospectivos con el fin de determinar su real aporte a este grupo de pacientes.

\section{BIBLIOGRAFÍA}

1. Jemal A, Sieger R, XU J, Ward R. Cancer statistic, 2010. CA Cancer J Clin 2010;60:277-300.

2. Kataria SK. The role of radiotherapy in ovarian cancer. Ind J Medical and Pediatric Oncol 2007;23:18-23.

3. Piver MS. Prophylactic Oophorectomy: Reducing the U.S. death rate from epithelial ovarian cancer. A continuing debate. The Oncologyst 1996;1:326-31.

4. Makatsosis T, Seiden MV. High dose therapy for ovarian carcinoma. The Oncologyst 1997;2:330-9.
5. Santini A, Torres M. Radioterapia posoperatoria en el cáncer de mama. Indicaciones, objetivos y resultados. En: Vázquez T \& Santini A. Los cánceres más frecuentes. Tomo 2. Oficina del libro. Montevideo, 2000.

6. Remberger K. Pathologische Anatomie der ovarialkarzinome und die kilinschen der malignant. In: Georgii A (ed). Aspekte der Kliniscen Onkologie. Verh Dtsch Krebsges 1984: 671-82.

7. Dvoretsky PM, Richards KA, Angel C, et als. Distribution of disease at autopsy in 100 women with ovarian cancer. Hum Pathol 1988;19:57-63.

8. Smith JP, Rutledge, Declos L: Prospective treatment of early cancer of ovary. A random trial between postoperative irradiation and chemotherapy. J Natl Cancer Inst 1975;42:149-53.

9. Mosalaei A, Kazerooni T. Results of post-operative abdomino-pelvic radiotherapy in intermediate- and high-risk epithelial ovarian carcinoma. Eur J Cancer Care (Eng) 2008;17(4):371-6.

10. Ozols RF, Rubin SC, Dembo A, Robboy S. Epitelial ovarian cancer. In: Hoskins W, Perez CA and Young R. Principles and Practice of Gynecologic Oncology. J.B. Lippincott Co. Philadelphia. 1997. p 731-80.

11. Dembo AJ. Abdominopelvic radiotherapy in ovarian cancer. A 10 year experience. Cancer 1985;55:2285-90.

12. Sedlaceck TV, Spyropoulus P, Cifaldi, et als. Whole abdomen radiation therapy as salvage treatment fot epithelial ovarian carcinoma. Cancer J Sci Am 1997;3:358-63.

13. Reinfuss M, Kojs Z, Skolyszewsky J. External beam radiotherapy in the management of ovarian carcinoma .Radiother Oncol 1993:26:26-32.

14. Hepp R, Baeza M, Olfos $P$, et als. Adjuvant whole abdominal radiotherapy in epithelial cancer of the ovary. Int J Radiat Oncol Biol Phys 2002;53;360-5.

15. Goldberg N, Peschel RE. Postoperatoive abdominopelvic radiation therapy for ovarian cancer. Int J Radiat Oncol Biol Phis 1988;14:425-9.

16. Dembo AJ. Radioterapeutic management of ovarian cancer. Sem Oncol 1984;11:238-50.

17. Fuller DB, Sauce WT, PlenK HP. Analysis of postoperative radiation therapy in stage I thorougt III epithelial ovarian carcinoma. J Clin Oncol 1987;5:897-905.

18. Petereit DG, Sharda NN, Hollister TC, et als. Adjuvant whole abdominal radiation for low risk and intermediate risk (Dembo clasification) ovarian cancer. Gynecol Oncol 1997;64:339(abstr 191).

19. Weiser EB, Burke TW, Heller PB, et als. Determinants of survival of patients with epithelial ovarian carcinoma following whole abdomen irradiation (WAR). Gynecol Oncol 1988;30:201-8.

20. Klaassen D, Shelly W, Starreveld A, et als. Early stage ovarian cancer. J Clin Oncol 1988;6:1254-63.

21. Smith JP, Rutledge FN, Declos L. Postoperative treatment of early cancer of the ovary: a randomized trial between postoperative irradiation an chemotherapy (monograph). J Natl Cancer Inst 1975;42:238-50.

22. Sell A, Bertelson K, Andersen JE. Randomized study of whole abdomen irradiation versus pelvic irradiation plus cyclophosphamide in treatment of early ovarian cancer. Gynecol Oncol 1990;37:367-73. 
23. Chiara S, Pierfranco C, Franzone $P$, et als. Hightrisk early-stage ovarian cancer: randomized clinical trial comparing cisplatin plus cyclophophamide versus whole abdominal radiotherapy. Am J Clin Oncol 1994;17:72-6.

24. Dowlatshahi M, Miller M, Semarad N, et als. Use of concomitant low dose cisplatinum and whole abdominal radiation (WARP) as salvage therapy for persistent ovarian carcinoma after positive second look precedure: a pilot study. Cancer J Sci Am 1999;5(abstr):123.

25. Bermudez RS, Rembert J, Hsu IC. Ovarian cancer, Chapter 31, In: Hansen EK \& Roach M III. Handbook of evidence based radiation Oncology. 2ed Springer, New York - Heidelberg - London 2010.

26. Muyala S, Wolfson A. ovarian and fallopian tube cancer. In: Lu J \& Brady L. Eds. Radiation Oncology. And Evidence Based Approach. Springer - Varlag. Berlin, Heidelberg 2008.

27. Ledermann JA, Dembo AJ, Sturgeon JF, et als. Outcome of patients with unfaborable optimally cytoreduced ovarian cancer treated with chemotherapy and wole abdominal radiation. Gynecol Oncol 1991;41:30-5.

28. Thomas GM. Is there a role for consolidation or salvage radiotherapy after chemotherapy in advanced epitelial ovarian cancer? Gynecol Oncol 1993;51:97-103.

29. Randall ME, Barrett RJ, Spirtos NM, et als. Chemotherapy, early surgical reassessment, and hyperfractionated abdominal radiotherapy in stage III ovarian cancer: Int J Radiat Oncol Biol Phiys 1994;27:169-76.

30. Goldhisch A, Greiner R, Dreher E, et als. Treatment of advanced ovarian cancer with surgery, chemotherapy, and consolidation of response by whole-abdominal radiotherapy. Cancer 1988;62:40-7.

31. Haie C, Pejovic-Lenfant MH, George H, et als. Whole abdominal irradiation following chemotherapy in patients with minimal residual disease after second look surgery in ovarian carcinoma. Int J Radiat Oncol Biol Phiys 1989;17:15-9.

32. Pikel H, Lahousen M, Petru E, et als. Consolidation radiotherapy after carboplatin-based chemotherapy in radically operated advanced ovarian cancer. Gynecol Oncol 1999;72:215-9.

33. Dinniwell, Lock M, Pintile M, et als. Consolidative abdominopelvic radiotherapy after surgery and carboplatin/paclitaxel chemotherapy for epithelial ovarian cancer Int J Radiat Oncol Biol Phys 2005;62(1):104-10.

34. Biete A, Valduvieco I, Rovirosa A, et als. Whole abdominal radiotherapy in ovarian cancer. Reports of practical oncology and radiotherapy 2010;15:27-30.

35. Reddy S, Lee MS, Yordan E, et als. Salvage whole abdominal irradiation therapy, its role in ovarian cancer. Int J Radiat Oncol Biol Phis 1993;27:879-84.

36. Baker K, Reddy S, Lee MS, et als. Salvage whole abdominal radiation therapy for ovarian cancer: A twelve year experience. Int J Radiat Oncol Biol Phys 1996;36(suppl 1):176.

37. Cmelak A, Cox RS, Kapp DS. Long term survival with whole abdomipelvic irradiation (WAI) in platinum-refractory ovarian cancer. Int J Radiat Oncol Biol Phys 1996;(suppl 1)36:176.

38. Eifel PJ, Gerschenson DM, Delclos L, et als. Twice daily, split course whole abdominal radiation therapy after chemotheray and positive second look laparotomy for epitelial ovarian carcinoma. Int J Radiat Oncol Biol Phys 1991;21:1013-8.

39. Fein D, Morgan L, Marcus R, et als. Stage III ovarian carcinoma, an analysis of treatment results and complications following hyperfractionated abdominal pelvic irradiation for salvag. Int J Radiat Oncol Biol Phys 1994;27:169-76.

40. Bolis G, Zanaboni F, Vanoli $P$, et als. The impact of whole abdomen radiotherapy on survival in advanced ovarian cancer patients with minimal residual disease after chemotherapy. Gynecol Oncol 1990;39:150-4.

41. Kuten A, Stein M, Steiner M, et als. Whole abdominal irradiation following chemotherapy in advanced ovarian carcinoma. Int J Radiat Onco Biol Phys 1988;14:273-9.

42. Scharay M, Martinez A, Howes A, et als. Advanced epithelial ovarian cancer: salvage whole abdominal irradiation for patients with recurrent or persistent disease after combination chemotherapy. $\mathrm{J}$ Clin Oncol 1988;6:1433-9.

43. Böttcher HD, Adamientz IA, Schopohl B. Radiation planing in female genital cancer. In: Vahrson H. Radiation oncology of gynecological cancers. Springer ed, Berlin 1997.

44. La Rouere J, Perez-Tamayo C, Fraass B, et als. Optimal coverage of peritoneal surface in whole abdominal radiation for ovarian neoplasma. Int J Radiat Oncol Biol Phys 1989;17:607-12.

45. Fuks Z. External radiotherapy of ovarian cancer: Standard approaches and new fronires. Semin Oncol 1975;2:253-9.

46. Martinez A, Schray MF, Howes AE, et als. Postoperative radiation therapy for epithelial ovarian cancer: the curative role based on 24-year experience .J Clin Oncol 1985;3:901-11.

47. Fazekas JT, Mayer JG. Irradiation of ovarian carcinomas. A prospective comparison of the opendfield and movin-strip techniques. Am $\mathrm{J}$ Roentgenol 1974;120:118-23

48. Dembo AJ, Bush RS, Beale FA, et als. A randomize clinical trial of moviong strip vs opend field whole abdominal irradiation in patients with invasive epithelial cancer of ovary (abstract). Int J Radiat Oncol Biol Phys 1983;9:97.

49. De Meerleer G, Vandecasteele K, Ost P, et als. Whole abdominopelvic radiotherapy using Intensity-Modulated Arc therapy in the palliative treatment of chemotherapy_resistant ovarian cancer with bulky peritoneal disease: a single-Institution experience. Int $\mathrm{J}$ Radiat Oncol Biol Phys 2011; 79:775-81.

50. Lanciano R,Reddy S, Corn B, et als. Update on the role of radiotherapy in ovarian cancer. Sem Oncol 1998;25:361-71.

51. May LE, Bellintson JL, Roland TA, et als. Paliative benefit of radiation therapy in advanced ovarian cancer. Gynecol Oncol 1990;37:408-11.

52. Corn BW, Lanciano RM, Boente M, et als. Recurrent ovarian cancer: Effective radiotherapeutic palliation after chemotherapy failure. Cancer 1994;74:2979-83.

53. Cardenes H, Randall M. Integrating radiation therapy 
in the curative mamagement of ovarian cancer: current issues and future directions, Sem Radiat Oncol 2000;10:61-70.

54. Schwarts PE, Rutherford TJ, Chambers JT, et als. Neoadyuvant chemotherapy for advanced ovarian cancer. Gyneco Oncol 1999;72:93-9.

55. Santini A, Torres M, Apardian R. Radioquimioterapia concomitante en el cáncer de cabeza y cuello: ¿un tratamiento estándar? Rev Med Uruguay 1998;14:106-9.

56. Silver DF, Wheeless CR, Dubin NH. Radiotherapy as a cisplatin-sensitizer in a resistant ovarian carcinoma cell line. Cancer 1996;77:1850-3.

57. King La, Downwy Go, Potish RA, et als. Concomitan whole abdominal radiation and intraperitoneal chemotherapy in advanced ovarian carcinoma: a pilot study. Cancer 1991;67:2867-71.

58. Rochet N, Sterzing F, Jensen A, et als. Intensity-Modulated Whole abdominal radiotherapy after surgery and carboplatin/taxane Chemotherapy for advanced ovarian cancer: Phase I Study. Int J Radiat Oncol Biol Phys 2010;76:5:1382-9. 